\title{
LENGTH-TO-LENGTH TRANSMISSION AS THE BASIS OF CONDUCTION, AND SOME RELATED CONSIDERATIONS ABOUT THE LAWS OF EXCITATION
}

\author{
KAZUMI YAMAGIWA * \\ Department of Physiology, Tokyo Medical and Dental University, Bunkyo-Ku, Tokyo
}

At one time I proposed (1) the idea of length-to-length conduction to replace the classical one of point-to-point conduction. What was actually meant was that, although conduction appears to be point-to-point or continuous, there must always exist a certain action length, which is long enough to excite another length. I pointed out then that some of the fundamental phenomena of conduction could be well understood from this viewpoint. While I was hesitating about its publication because of an unsatisfactory assumption made, Rushton's paper (2) appeared, which, although quite different from mine in the way of approach and in the subjects treated, agreed with mine in the most important conclusion that at least a certain minimum fibre length must be activated for the initiation of nervous impulse. Encouraged by Rushton's paper, I published mine with defect and all.

The present paper is an improvement of and a supplement to the old paper. The mathematical part was renewed and simplified. The greatest addition made is Chapter V, where I attempted to explain some of the fundamental laws of excitation from the same viewpoint.

\section{AN ACTION LENGTH AS THE SOURCE OF ANOTHER ACTION LENGTH (CALCULATION)}

The object here is to calculate the action length (briefly, A.L.) $y$ which can be excited by a given A.L. $x$ (see fig. 1). Actually, the excitation in $y$ begins at its left end, to proceed to the right. The action current from these early activated portions will co-operate with that of $x$, to enlarge $y$. But here this effect is put aside, and the length $y$ which is excited by $x$ and $x$ only by $t_{m}$ (the moment of maximal stimulating effect) is considered.

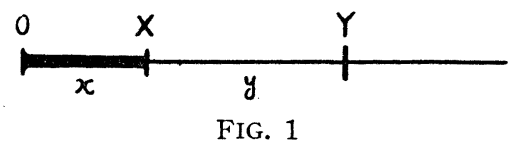

$x$ : excited region, $y$ : resting region, $Y$ : point being stimulated by threshold.

Received for publication March 28, 1955.

*山極一三 
Let now the peak potential at $X$ and $Y$ be $P_{X}$ and $P_{Y}$ respectively. Then we have

$$
P_{Y}=P_{X} e^{-y / k}=S
$$

$k$ : Length constant $=\sqrt{\frac{r_{m}}{r_{i}+r_{e}}}$, where $r_{m}, r_{i}$ and $r_{e}$ are respectively the membrane, internal and external resistances of unit length of fibre,

$S$ : Threshold (in terms of peak voltage).

$P_{x}$ will be expressed approximately by

$$
\begin{aligned}
& P_{X}=\int_{0}^{x} V e^{-x / k^{\prime}} d x=-V k^{\prime}\left[e^{-x / k^{\prime}}\right]_{0}^{x}=V k^{\prime}\left(1-e^{-x / k^{\prime}}\right) \\
& V: \text { Action potential (taken constant), } \\
& k^{\prime}: \text { Length constant of active fibre }=\sqrt{\frac{r^{\prime} m}{r_{i}+r_{e}}} .
\end{aligned}
$$

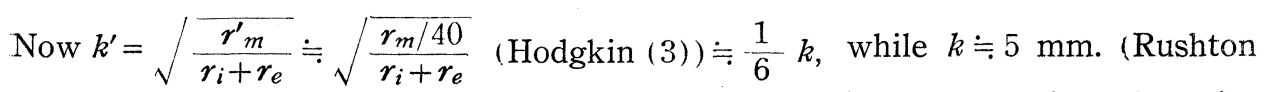
(4)). Hence we can assume $k^{\prime} \fallingdotseq 1 \mathrm{~mm}$. This relation will be approximately maintained as long as $k$ does not vary very greatly.

Thus we have in place of equation (2),

$$
P_{X}=V\left(1-e^{-x}\right), \quad x \text { in } \mathrm{mm} .
$$

This result is quite similar to that obtained recently in the case of myelinated nerve fibres (Yamagiwa (5)).

Now, putting eq. (3) into (1), we have

or

$$
\begin{gathered}
P_{Y}=V\left(1-e^{-x}\right) \cdot e^{-y / k}=S, \\
e^{-y / k}=\frac{S}{V} \frac{1}{\left(1-e^{-x}\right)} \\
y=k \log \frac{V}{S}\left(1-e^{-x}\right)=k \log (\text { Safety factor })\left(1-e^{-x}\right) \\
=k\left\{\log (\text { S.F. })+\log \left(1-e^{-x}\right)\right\}
\end{gathered}
$$

Fig. 2 is the general graphical representation of eq. (4). The position of the $x$-axis is determined by the value of the safety factor. It should be noticed here that the safety factor defined as $V / S$ is the safety factor for a local excitation and not for a propagated impulse. The exact shape of the graph and the exact values of $l, L$ and $M$ will be given in Chapter VI.

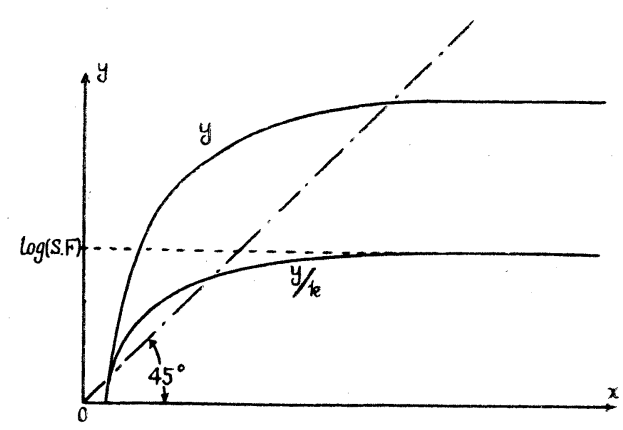

FIG. 2. $y$-x-relation in general. 


\section{THE GENERAL MEANING OF THE MATHEMATICAL RESULT}

According to the values of $k$ and safety factor, there can be three types of $y$ - $x$-relation (fig. $3, \mathrm{I}, \mathrm{II}, \mathrm{III}$ ). After all, there can be four cases, namely
1) $y=o$,
2) $y=x$,
3) $y<x$, and
4) $y>x$.

Considering simply that $x$ produces $y_{1}$, and $y_{1(2)}$ produces $y_{2(3)}$ successively, we can have a rough picture of A.L.-variation in course of conduction. However, the length $y$ thus obtained is, as mentioned above, not the length which will actually be excited by the moment, $t_{m}$. The latter, $y^{\prime}$, must be somewhat larger than $y$, as follows:

$$
y^{\prime}=y+\Delta y
$$

$\Delta y$ must be a function of $y$, accordingly, of $x$, but it is easily seen that there will practically be an upper limit in the value of $\Delta y$, hence, roughly

$$
\Delta y=f(x)=a\left(1-e^{-b x}\right), \quad a, b: \text { constants. }
$$

Hence, even if $y$ is replaced by $y^{\prime}$ in fig. 2-3, there occurs no essential difference except that the ordinate increases its value in the region of small $x$. The three types of $y$-x -relation will invariably appear, and as this is the very point to be discussed, $y$ will be taken up for $y^{\prime}$, for simplicity, in the present argument. We shall express, for example, the condution velocity $v$ by $v=y / t_{m}$, instead of by $y^{\prime} / t_{m}$.

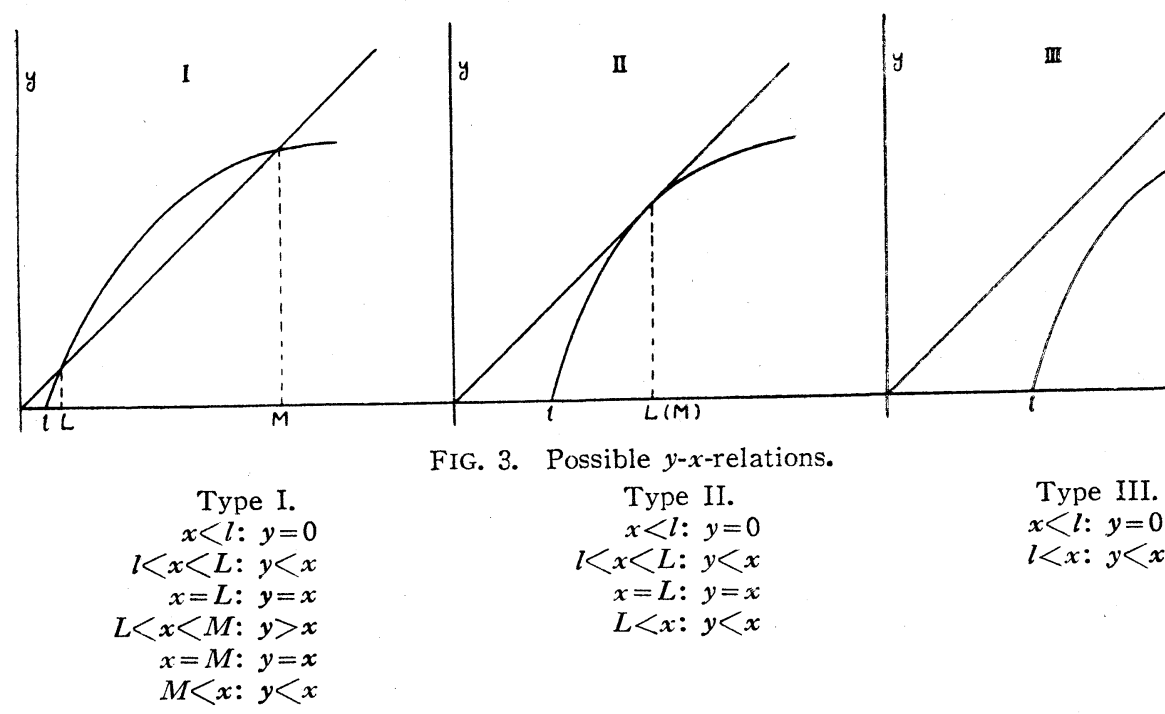

III. THE MODE OF CONDUCTION IN RELATION TO THE INITIAL ACTION LENGTH

Suppose that $x$ is the initial action length given, and that it produces $y_{1}, y_{2} \ldots$ successively, satisfying the mathematical relation of eq. (3) between $x$ and $y_{1}$, 
$y_{1}$ and $y_{2}$ and so on. The variation of $y$ in the course of conduction will then. be as follows:

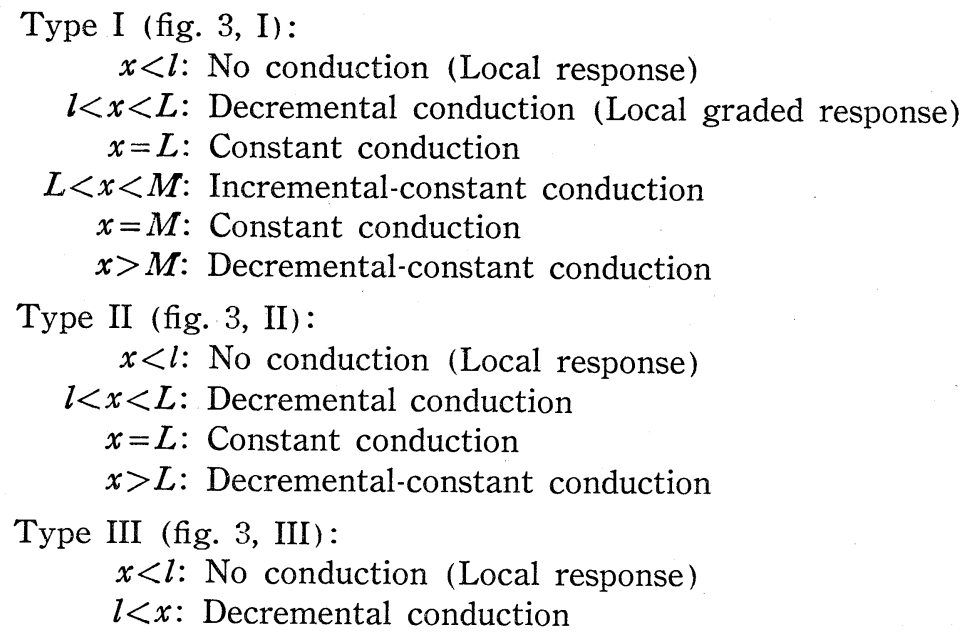

If the cases $x=L$ and $x=M$ are excepted as special cases, there are at least three modes of conduction, i.e.

1) Decremental type,

2) Incremental-constant type, and

3) Decremental-constant type.

Of these three, the third one is the case where $x>M$ (in type I) or $x>L$ (in type II), both of which will be very rare. So, we have perhaps two modes after all, that is, the decremental and the incremental-constant types. And in the incremental-constant type, the action length, if observed at a long distance, will always be caught as constant, without regard to the initial action length, that is, to the stimulus intensity applied. It will be shown later (Chapter VI) that this is the normal type. The above statement concerns the action length, but the same can be said about the velocity and the action potential, because the velocity can relatively be treated as $v \fallingdotseq$ A.L. $/ t_{m}$ and the potential on the fibre surface increases with the action length (2).

One of the most important results is the appearance of the specific length $L$, which is needed for the initiation of impulse. This plays the most important role throughout the present argument. This is identical with Rushton's minimal length (2), which is said to be as long as $0.86 \mathrm{~mm}$. The length appears very much shorter in the present theory, as will be shown in Chapter VI.

\section{SEVERAL EXPLAINABLE CONDUCTION PHENOMENA}

\section{Conduction in a narcotized nerve}

Let us first quote eq. (4):

$$
y=k\left\{\log (\mathrm{S} . \mathrm{F} .)+\log \left(1-e^{-x}\right)\right\}, \quad \text { S.F. }=\frac{V}{S}, \quad k=\sqrt{\frac{r_{m}}{r_{e}+r_{i}}} .
$$




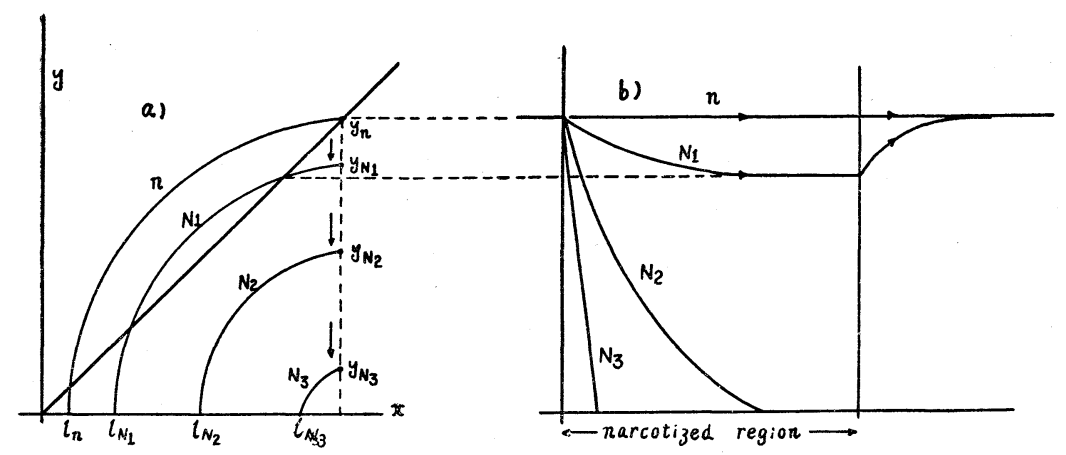

FIG. 4. Variation of A.L. in narcotized region.

The way physiological constants vary with narcosis is the clue by which the mode of conduction in a narcotized nerve can be judged from the present point of view.

The change of $r_{m}$ is said to be very little (Tasaki $(6-8)$ ), so that the value of $k$ will remain approximately constant. On the other hand, $V$ decreases and $S$ perhaps increases, hence, after all, the safety factor will decrease. As a consequence, the $y$-x-relation will change its type from fig. 3 , I to fig. 3 , II and III gradually with the depth of narcosis (fig. $4, a$ ). The A.L. which was $y_{n}$ in the normal region, will drop to $y_{N_{1}}$ or $y_{N_{2(3)}}$ at the entrance and proceeds on decremental-constantly or simply decrementally or stops there to give a local response, according to the grade of narcosis (fig. $4, b$ ). This can be shown graphically by fig. 5 , where the supposed changes of the first A.L., $L$ and $l$ are illustrated as functions of the depth of narcosis (about $L$ and $l$, see fig. 3).

If, in fig. 5 , the depth of narcosis is between zero and $N_{L}$, then we have a decremental-constant conduction. If it is between $N_{L}$ and $N_{l}$, we have a decremental conduction, and if the narcosis is deeper than $N_{l}$, then we have just a

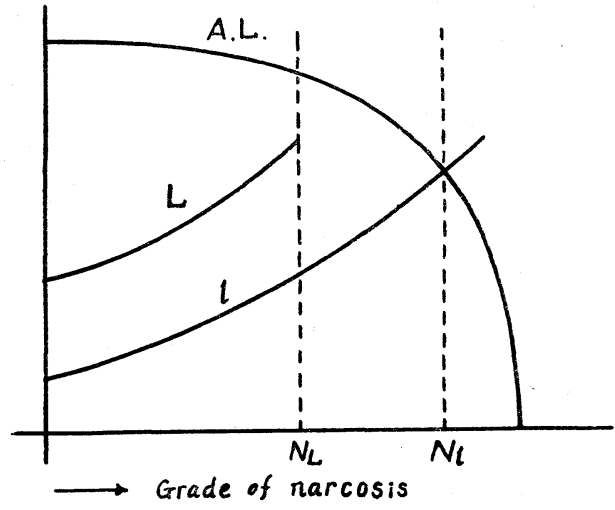

FIG. 5. A.L., $L$ and $l$ in relation to the grade of narcosis (supposed). local response at the entrance. The point $N_{L}$ indicatest he critical depth of narcosis, at which the $y$ - $x$-curve just touches the $45^{\circ}$-straight line.

The critical length to be narcotized for impulse blocking can be calculated in the following way. In fig. 6 ,

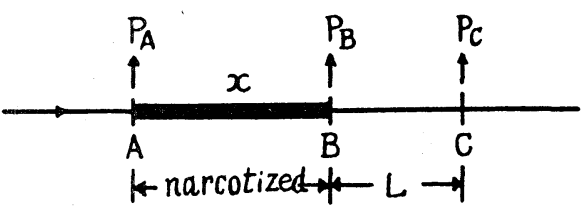

FIG. 6. Critical length for blocking impulse. $P_{A}, P_{B}, P_{C}$ : Potetials at $A, B$ and $C$. 
$P_{A}$ : Potential at the left edge $(A)$ of narcotized region.

$P_{B}$ : Potential at the left edge $(B)$ of normal region.

$P_{C}$ : Potential at $C(B C=L)$.

Then, approximately

$P_{B}=P_{A} e^{-x / k_{N}}, \quad \dot{k}_{N}$ : Length constant of narcotized fibre.

$P_{C}=P_{B} e^{-L / k}, \quad k$ : Length constant of normal fibre.

The condition necessary for blocking is $P_{C} \leqq S$.

Hence,

$$
\begin{gathered}
P_{C}=P_{B} e^{-L / k}=P_{d} e^{-x / k_{N}} \cdot e^{-L / k}=P_{A} e^{-\left[\left(x / k_{N}\right)+(L / k)\right]} \leqq S \\
e^{-\left[\left(x / k_{N}\right)+(L / k)\right]} \leqq S / P_{A} \\
x / k_{N}+L / k \supseteqq \log P_{A} / S=\log \text { (Safety factor). } \\
\therefore \quad x \geqq k_{N} \log (\mathrm{S} . \mathrm{F} .)-\left(k_{N} / k\right) \cdot L . \\
k_{N} \fallingdotseq k: x \supseteqq k \log (\mathrm{S} . \mathrm{F} .)-L
\end{gathered}
$$

If we employ Rushton's data, $k \fallingdotseq 5 \mathrm{~mm}$. (4), S.F $\fallingdotseq 3$ (2), $L \fallingdotseq 0.8 \mathrm{~mm}$. (2), we have $x \geqq 4.2 \mathrm{~mm}$.

If $L$ is $0.1-0.2 \mathrm{~mm}$. at the largest (see Chapter VI), then, neglecting it, we have $x \supseteqq 5 \mathrm{~mm}$. It may be a little larger, because the safety factor seems to be a little larger than assumed, but still it is just about the same value as obtained experimentally long ago (Lucas, Kato, Tasaki $(6,9,10,11)$ ).

\section{Temperature and conduction velocity}

It is well known that the conduction velocity increases and decreases according to temperature. From the present theory the change of velocity due to temperature can be judged, although qualitatively, from the following equation.

$$
\begin{aligned}
v \fallingdotseq \frac{y}{t_{m}}=\frac{k}{t_{m}} & \left\{\log (\mathrm{S} . \mathrm{F} .)+\log \left(1-e^{-x}\right)\right\} \\
k & =\sqrt{\frac{r_{m}}{r_{i}+r_{e}}}, \quad t_{m}: \text { moment of max. stim. }
\end{aligned}
$$

Just how the resistances $\boldsymbol{r}_{m}, \boldsymbol{r}_{i}$ and $\boldsymbol{r}_{\boldsymbol{e}}$ are affected by temperature is not quite clear, but presumably all in the same way and in the same magnitude. Therefore, the value of $k$ will remain approximately constant, while the safety factor will suffer a great alteration, because in high temperature $V$ gets larger (Hodgkin (12)) and $S$ gets perhaps smaller. The $v$-x-relation will thus change in the direction from type III to II or I (fig. 3) when the temperature is raised.

We can thus understand that 1) the conduction velocity gets generally larger in high temperature, and 2) the velocity change in relation to the conduction distance can vary from the decremental type to the constant type.

3. Conduction velocity in relation to stimulation intensity and to conduction distance.

Of the $v$-x-relation expressed by eq. (5), there can be three types according to the quantitative differences in physiological constants, just as in the case of action length. And again in each type, there can occur a local response, decre- 
mental conduction or constant conduction, according to the initial action length, that is, to the stimulus intensity applied. Thus we expect $v$ to be a function of both stimulus and distance.

Whether or not the conduction velocity varies with the stimulus intensity or with the conduction distance, has often been a subject of investigation, and it seems, since Engelmann (13) and Nicolai (14), to be generally believed that the velocity is constant throughout, being indifferent to these factors. Recently Tasaki (15) beautifully demonstrated constancy with his single fibere prepara tion.

However, constancy can also be expected from the present theory, as long as the observation is made at a remote region. A question lies in the direct vicinity of the stimulated site, where the wave can develop very quickly, to attain its final value very soon. Precise examinations are left for the future.

\section{Fibre size and conduction velocity}

In eq. (5), we can put $k=\sqrt{\frac{r_{m}}{r_{i}+r_{e}}} \fallingdotseq \sqrt{\frac{r_{m}}{r_{i}}}$, if a fibre in normal condition is considered. Then

Hence

$$
\begin{gathered}
r_{m}=C_{1} \frac{1}{R}, \quad r_{i}=C_{2} \frac{1}{R^{2}}, \quad R: \text { radius, } \\
k=\sqrt{\frac{C_{1}}{C_{2}} R}=C_{3} \sqrt{R} .
\end{gathered}
$$

Hence, $k$ will increase in proportion to $\sqrt{R}$ in thicker fibres. On the other hand, $V$ remains constant and perhaps $S$ does so, too, because there is no reason for $S$-change in this case, although the usual threshold can decrease. So, the safety factor will be unchanged. Thus we see that in thicker fibres velocity can increase approximately in proportion to $\sqrt{R}$. In addition, secondary acceleration will occur in succession as the result of increased $y$ in the initial stage, so that the mean $v$ will be larger than proportional to $\sqrt{R}$.

\section{Electrotonus and conduction velocity}

It is well known that $v$ is large in the cathodic region and small in the anodic region. Now,

$$
v \fallingdotseq \frac{y}{t_{m}}=\frac{k}{t_{m}} \log (\text { S.F. })\left(1-e^{-x}\right), \quad k=\sqrt{\frac{r_{m}}{r_{i}+r_{e}}}, \quad \text { S.F. }=\frac{V}{S} .
$$

How the electrotonus affects $k$ and S.F. is the clue for judgment.

The variation of $k$ under electrotonus is unknown, but presumably it decreases under catelectrotonus, because $r_{m}$ will decrease, which means that the $y(v)$-x-relation will change in the direction from fig. 3, I-towards II- or III- type. The variation of S.F. is also unknown, because the variation of $S$ is unknown, although $V$ is known to decrease under catelectrotonus. On the other hand, however, it is also known experimentally that the usual threshold, that is, the 
threshold for initiating an impulse, is low under the cathode, which points, if viewed from the presnt theory, to a smaller value of $L$. And, the smaller value of $L$, in spite of the decrease of $k$ and $V$, suggests strongly that $S$ must have become sufficiently small to make $V / S$ sufficiently large to over-compensate the contrary effect of $k$ and to alter finally the type of $y(v)$-x-relation from III or II towards I. Thus, $v$ will become larger under catelectrotonus in spite of smaller $V$. The state of affairs will be just the opposite under anelectrotonus. In connection with this, it is very suggestive that in a long Lillie's nerve model, the middle part of which is anodically (that is, cathodically in physiological sense) polarized, the wave passes over the middle part easily with an enlarged action length and velocity, while, if cathodically polarized, the action length is shortened and the velocity is slowed so much that the wave eventually stops at the middle or a short way this side of it. In case it passes over, it regains the original size of the action length and of the velocity very quickly (Yamagiwa (16)).

As to the cathodic depression or block, a somewhat different consideration seems to be needed, which I shall describe later.

\section{Graded and local responses}

It has been confirmed by many investigators that in muscle, local contractions of various magnitudes, non-conductive or only locally conductive, can occur according to the electrode size or to the stimulation intensity, and further that if the electrode size or the stimulation intensity attains a certain critical value, the contraction changes suddenly into one which is conducted along the whole length (17-25). As to the reason of the graded response, Gelfan et al. imagined a minute area which is not capable of exciting the adjacent region, but did not explain how conduction of an all-or-nothing character takes place when the stimulus attains a certain definite level. Asmussen supposed that a stimulus, if intensified to a certain extent, will stimulate a muscle end-plate and thus bring forth the same result as that obtainable by nerve stimulation.

Recently, the local response of nerve fibre, too, has been reported one after another (26-35), and thus the so-called local response seems to have been firmly established both in muscle and nerve. However, the interpretation is divided as to its relation to the spike:

1) The identity theory,

a) Locality difference theory or minute area theory,

b) Intensity difference theory or subthreshold activity theory,

2) Non-identity theory.

The first two opinions regard the local response as identical with the spike, the only difference being in a) that it arises from a minute area, which is itself allor-nothing (Hodgkin et al. (25-27)), and in b) that it is an expression of a subthreshold excitation of one and the same locality (Cole, Hodgkin, (34), (58-59)). This is the most prevailing at present. The third opinion, expressed by Rosenblueth (36), takes the local response as quite different from the spike in nature as well as in properties.

These opinions will be criticized in "Discussion." Here I will take the first 
opinion as correct, and show how simply the known phenomena can be understood from this stand-point.

Take the type I (fig. 3 ) as the representative of possible $y$-x -relations. Then, as stated already, the response can be strictly local, decrementally conductive, or finally, constantly conductive, according to the magnitude of $x$ initially given, i.e., to the stimulation intensity applied. This state of affairs is just the same in the cases of type II and III, in the sense that the local and graded responses can similarly take place, if not all-or-none conduction. In this way, we can very simply explain the occurences of local response, local graded response and the all-or-nothing response as the natural sequence of stimulation intensity, weak or strong.

In Rushton's theory, as in mine, a certain minimal length is required as necessary for initiation of the impulse, but the event that can happen in his is either local or wholely conductive, the intermediate graded response having no space to come in. This is one of the differences between his theory and mine.

\section{RE-CONSIDERATION OF SOME FUNDAMENTAL LAWS OF EXCITATION}

We have quite a number of investigations, experimental as well as theoretical, about the laws of excitation. Thereby, the index of excitation employed has always been the conducted response. In recent years, with the introduction of delicate techniques, the response of a very minute area, for example, a single node of Ranvier, has been taken as an index, but it has been the so-called fullsized spike that was taken up, so it was equivalent to taking the propagated disturbance as the index. In theoretical treatment, however, it was always the excitation of the stimulated point, that was considered. In other words, the propagated impulse was assumed to be identical with the local excitation, which, I think, might have been a source of confusion. The same can be said about the "threshold" of stimulus: the threshold for propagation should be strictly distinguished from that for local responses, and the threshold of a local response of a certain magnitude should again be distinguished from the threshold of another local response of a different magnitude.

Now, if there is really a certain minimal length to be excited for initiation of an impulse, then it must have always been excited directly in the experimental investigations of excitation laws hitherto made. The laws thus derived are laws for exciting the length in question simultaneously, but cannot always be applied to the excitation of the stimulated point. From this viewpoint I shall reexamine some classical works, to draw out something new, if possible.

\section{The rectangular pulse (Appearance of $V_{r}$ and $t_{r}$ )}

Most characteristic in this field of investigation is the appearance of the rheobase $V_{r}$ and its utilization time $t_{r}$. The existence of $V_{r}$ seems very probable but that of $t_{r}$ is a matter not always easy to understand. Ozorio de Almeida (37) and Hill (38) succeeded in explaining it, but in de Almeida's theory, the basic assumption adopted is questionable, and in Hill's theory, there is a new concept "accommodation" introduced. I will show here that we can understand $V_{r}$ and $t_{r}$ quite easily, without the help of any new concept. 
In fig. 7 , the point $x=0$ and $x=L$ denote respectively the so-called stimulated point and the point of the minimal length, $L$. (The point $x=L$ was taken for simplifying the description, although, strictly, we should take the point $x=L / 2$ ). Let the voltage applied at $x=0$ be $V_{0}$, then the voltage at $x=L$ will be

$$
\begin{gathered}
V_{L}=V_{0} e^{-L / k}=\frac{1}{m} V_{0} \\
k=\text { length const, } \frac{1}{m}=e^{-L / k}<1, \quad m>1 .
\end{gathered}
$$

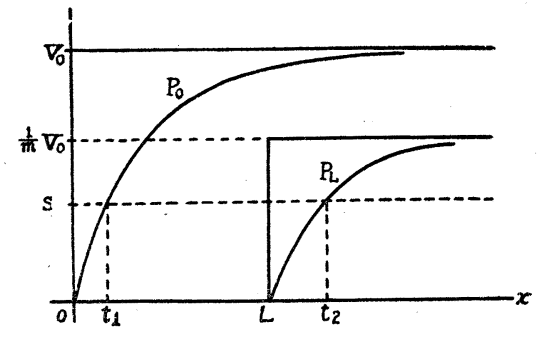

FIG. 7. Charging up of "local potential" at points $x=0$ and $x=L$, by direct current.

The local potential, $P$, is simply assumed to follow the well-known and often discussed $(39-40)$ equation

$$
P=V\left(1-e^{-t / \alpha}\right),
$$

$t$ : time, $\alpha$ : time constant.

Then,

$$
\text { at } \begin{aligned}
& x=0: P_{0}=V_{0}\left(1-e^{-t / \alpha}\right), \\
x & =L: P_{L}=\frac{V_{0}}{m}\left(1-e^{-t / \alpha}\right) .
\end{aligned}
$$

The excitation will occur at $P=S=$ const., so

Similarly

$$
\text { at } \begin{array}{r}
x=0: V_{0}\left(1-e^{-t_{1} / \alpha}\right)=S, \\
t_{1}=\alpha \log \frac{V_{0}}{V_{0}-S}
\end{array}
$$

$$
\text { at } x=L: t_{2}=\alpha \log \frac{V_{0}}{V_{0}-m S}
$$

$$
\therefore t_{2}-t_{1}=\Delta t=\alpha \log \frac{V_{0}-S}{V_{0}-m S}
$$

$\Delta t$ is zero when $V_{0}=\infty$, then increases with a decrease of $V_{0}$ to attain $\infty$ at $V_{0}=m S$. $t_{2}$ is nothing but the necessary duration being explored in the experiment.

Now, it is our basic assumption that the minimal length $L$ must be excited simultaneously for initiation of impulse, which means

$$
\Delta \mathrm{t} \leqq D, \quad D \text { : duration of excitation (at } x=0) \text {. }
$$

In the limiting case,

$$
\begin{gathered}
\alpha \log \frac{V_{0}-S}{V_{0}-m S}=D, \\
\frac{V_{0}-S}{V_{0}-m S}=e^{D / \alpha}, \\
V_{0}-S=e^{D / \alpha}\left(V_{0}-m S\right), \\
V_{0}\left(e^{/ D / \alpha}-1\right)=S\left(m e^{D / \alpha}-1\right),
\end{gathered}
$$




$$
V_{0}=S \frac{m e^{D / \alpha}-1}{e^{D / \alpha}-1} \quad(\text { a definite value })
$$

The value of $V_{0}$ calculated above will appear as $V_{r}$ in the experiment, because any voltage smaller than this is ineffective for initiating a propagated impulse, although it may be effective in provoking excitation at $x=0$ or even at $x=L$, if separated. The necessary duration, $t_{2}$, for $V_{r}$ will necessarily appear as $t_{r}$, the so-called "Hauptnutzzeit." Thus we see that the so-called rheobass $\left(V_{r}\right)$ is the liminal applied voltage for obtaining a propagated impulse, and $t_{r}$ the utilization time of $\frac{1}{m} V_{r}$ for excitation at the point $x=L$.

The above argument started from eq. (5), which gives $V_{r}$ for $t=\infty$, but no $t_{r}$. It is note-worthy that $t_{r}$ can appear from this equation if only a propagated impulse is taken as the index of excitation.

Let us show two other examples:

1) Nernst's formula

$$
i=\frac{a}{\sqrt{t}} \text { or } i^{2} t=a^{2}
$$

This formula involves no $i_{r}$, much less $t_{r}$.

Now,

$$
\begin{aligned}
& \text { at } x=0: i=i_{0} ; i_{0}^{2} t_{1}=a^{2} ; t_{1}=\frac{a^{2}}{i_{0}^{2}} \\
& \text { at } x=L: i=\frac{1}{m} i_{0} ; \frac{i_{0}^{2}}{m^{2}} t_{2}=a^{2} ; t_{2}=\frac{a^{2} m^{2}}{i_{0}{ }^{2}} \\
& \quad t_{2}-t_{1}=\Delta t=\frac{a^{2}}{i_{0}^{2}}\left(m^{2}-1\right) .
\end{aligned}
$$

In order that propagation can occur,

$$
\begin{aligned}
\Delta t & =\frac{a^{2}}{i_{0}{ }^{2}}\left(m^{2}-1\right) \leqq D \\
i_{0} & \equiv \sqrt{\frac{a^{2}\left(m^{2}-1\right)}{D}}, \\
\therefore \quad i_{r} & =\sqrt{\frac{a^{2}\left(m^{2}-1\right)}{D}}, \\
t_{r} & =\frac{a^{2} m^{2}}{i_{r}^{2}}=\frac{m^{2} D}{m^{2}-1} .
\end{aligned}
$$

2) Weiss formula

$$
i=\frac{a}{t}+b ; t=\frac{a}{i-b} \text {. }
$$

This empirical formula indicates simply that there is a particular value of $i$ for a particular value of $t$, experimentally. If there is $i_{r}$ actually, then there will be $t_{r}$. In this sense, Weiss's formula may be said to involve $i_{r}$ and $t_{r}$. But if we regard it as a theoretical formula, then we have $i_{r}=b$ as a limiting value 
for $t=\infty$, that is, the formula does not involve $t_{r}$. But still we can derive $t_{r}$ therefrom in the following way.

$$
\begin{gathered}
\text { At } x=0: t_{1}=\frac{a}{i_{0}-b}, \\
x=L: t_{2}=\frac{m a}{i_{0}-m b}, \\
\Delta t=t_{2}-t_{1}=\frac{m a\left(i_{0}-b\right)-a\left(i_{0}-m b\right)}{\left(i_{0}-m b\right)\left(i_{0}-b\right)}=\frac{a i_{0}(m-1)}{\left(i_{0}-m b\right)\left(i_{0}-b\right)} \\
=\frac{a(m-1)}{i_{0}\left\{1-\left(m b / i_{0}\right)\right\}\left\{1-\left(b / i_{0}\right)\right\}}
\end{gathered}
$$

Hence, $\Delta t$ is zero at $i_{0}=\infty$, and gets larger with a decrease of $i_{0}$ to attain $\infty$ at $i_{0}=m b$. There will be a certain value of $i_{0}$ which will give $\Delta t=D$. This will appear as $i_{r}$ experimentally, and its utilization time as $t_{r}$.

The above treatment tells us nothing about the correct law of excitation, but shows that $V_{r}$ and $t_{r}$ are invariably deduced from several excitation laws, if only the conducted impulse is employed as the index. This gives us an impression that $V_{r}$ and $t_{r}$, at least $t_{r}$, might be characteristics of the laws of conducted impulses, but not always of local excitation.

\section{The linearly progressive current (Appearance of the minimal gradient)}

Long ago, it was confirmed by Lucas (41) and Fabre (42) that the final necessary potential of linearly progressive currents gets higher and higher and finally reaches infinity with a decreasing gradient of current. The liminal gradient, that is, the smallest effective gradient was taken as a characteristic of each excitable tissue, and, as an appropriate expression of it, Lucas took the ratio gradient $/ i_{r}$ and called it the minimal gradient. Later, Fabre adopted the inverse value and named it the constante linéaire. This phenomenon makes a basis for the concept "accommodation" which was fully developed by Hill (38).

Now, from the present writer's viewpoint, the appearance of the minimal gradient can be quite simply understood as a characteristic quantity defined definitely by some physiological constants:

In fig. $8, S$ denotes the threshold potential, determined previously by a long rectangular pulse. Suppose that a linearly progressive current is changed into

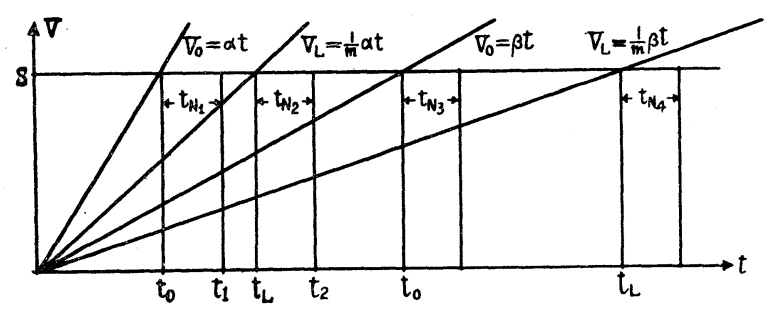

FIG. 8. Linearly progressive currents at points $x=0$ and $x=L$.

$S$ : Threshold. 
a constant current at the level of $S$. Now, let us assume simply that excitation occurs if the current flows on for a certain period $\Delta t_{N}$. This is an assumption quite free from the idea of "accommodation."

Let a current $V_{0}=\alpha t$ be applied at $x=0, \alpha$ being the gradient. Excitation will take place at $t_{1}=t_{0}+\Delta t_{N_{1}}$ at $x=0$, and at $t_{2}=t_{L}+\Delta t_{N_{2}}$ at the point $x=L$, where a voltage $V_{L}=\frac{1}{m} \alpha t$ becomes effective. For propagation to occur,

$$
t_{2}-t_{1}=\Delta t=t_{L}-t_{0}+\left(\Delta t_{N_{2}}-\Delta t_{N_{1}}\right) \leqq D .
$$

Generally, $\Delta t_{N_{1}}$ will be different from $\Delta t_{N_{2}}$, but $L$ is very small (see p. 152), so that $V_{0}$ and $V_{L}$ and, consequently, $\Delta t_{N_{1}}$ and $\Delta t_{N_{2}}$ will always be very near each other. So, neglecting the term $\left(\Delta t_{N_{2}}-\Delta t_{N_{1}}\right)$, we have approximately

$$
\Delta t=t_{L}-t_{0} \leqq D,
$$

From $t_{0}=\frac{S}{\alpha}$ and $t_{L}=\frac{m S}{\alpha}$, we have

$$
\begin{aligned}
& \frac{m S}{\alpha}-\frac{S}{\alpha} \leqq D . \\
& \alpha \geqq \frac{S(m-1)}{D},
\end{aligned}
$$

$S:$ Threshold voltage, $D$ : Duration of excitation, $m: e^{k / L}, \quad L:$ Minimal length, $k$ : Length constant.

The limiting case $\alpha=\frac{S(m-1)}{D}$ makes the minimal gradient definitely defined by physiological constants. According to Tasaki (43), $\alpha$ gets distinctly smaller

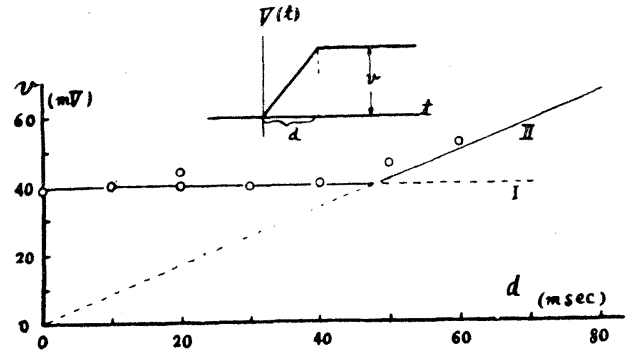

FIG. 9. Threshold voltage of linearly progressive current (Tasaki. Jap. J. Physiol. 1: $1,1950$. in lower temperature, while $S$ remains unchanged, a fact well understandable from the equation derived above, as due to the lengthening of $D$.

As a satisfactory answer to the above expectation, there is a very interesting experiment made by $\mathrm{Ta}$ saki (44) on a single node of Ranvier (fig. 9). Another similar one was added recently by Diecke (61). Tasaki attributes the gradual slope of Lucas to complications of the preparation, and Diecke to distortion of the stimulating current partly due to the sheath.

Tasaki's and Diecke's works above quoted indicate two important things, namely, 1) no threshold rise (in the horizontal part) and 2) almost sudden appearance of the minimal gradient. The first fact shows directly that there is no accommodation. The second fact was taken by Tasaki as expressing a necessary condition for provoking an excitation in general, that is, a condition for satisfying the relation $\frac{d E_{s}}{d t}>m$, where $E_{s}$ is the excitatory state as he calls 
it, and $m$ a certain definite number (45). But if interpreted from my viewpoint, the minimal gradient is the expression of a very simple fact that a current with a smaller gradient can not start an impulse, although it may elicit a local excitation.

Another interesting instance is Sugi's work (45). He carried out an investigation similar to that of Tasaki above quoted, on the sartorius muscle, employing his method of separating wall. He found that the muscle never failed to respond at a certain definite level of voltage, without regard to the gradient. Even the minimal gradient did not appear, as far as examined. Possibly, the excitation duration, $D$, might have been very long as he has claimed for a long time, so that the condition $\Delta t \leqq D$ could be maintained throughout. Sugi's method is similar to Tasaki's in principle, and it is note-worthy that the results above described were obtained in these special methods only.

3. The exponentially increasing current (Linear relationship between final voltage and time constant)

The exponentially increasing current is expressed by the following equation:

$$
V=V_{\infty}\left(1-e^{-t / \alpha}\right)
$$

This current has been often employed as a means for studying the phenomenon

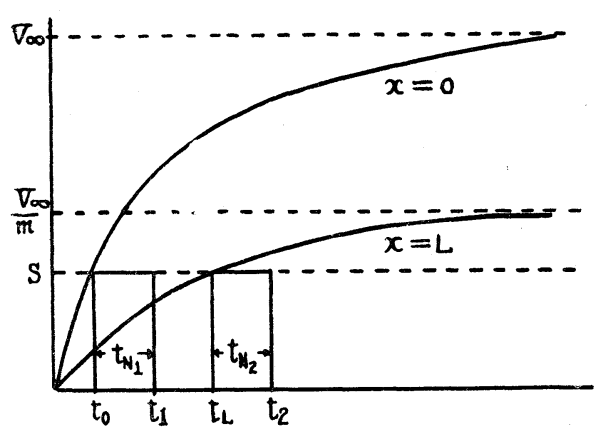

FIG. 10. Exponentially increasing current at points $X=0$ and $X=D$. $t_{N}$ : necessary duration. of "Einschleichen." Let us assume that 1) the potential level necessary for local excitation is independent of the rate of increase and 2) the current has to flow on for a little while $\Delta t_{N}$ (fig. $10)$, as in the case of linearly rising current. We lack experimental proof, but Sugi (46) observed in the work above quoted that the muscle responded always at an almost constant level of potential, without regard to the rate of increase. So, the above assumptions may perhaps be correct.

Now, the most firmly established fact in this field is the linear relationship

between $\frac{V_{\infty}}{S}$ and $\alpha$. We will show that this relation can very easily be derived from the present theory.

In order to have a propagated impulse, we have to have

$$
\Delta t=t_{2}-t_{1}=\left(t_{L}+\Delta t_{N_{2}}\right)-\left(t_{0}+\Delta t_{N_{1}}\right)=\left(t_{L}-t_{0}\right)+\left(\Delta t_{N_{2}}-\Delta t_{N_{1}}\right) \leqq D
$$

Neglecting the term $\left(\Delta t_{N_{2}}-\Delta t_{N_{1}}\right)$ for the same reason as before, we have

$$
\Delta t=t_{L}-t_{0} \leqq D .
$$

$t_{0}$ can be obtained from the equation $V_{\infty}\left(1-e^{-t_{0} / \alpha}\right)=S$, 
namely

$$
\begin{aligned}
t_{0} & =\alpha \log \frac{V_{\infty}}{V_{\infty}-S} . \\
t_{L} & =\alpha \log \frac{V_{\infty}}{V_{\infty}-m S}, \\
\therefore \Delta t & =t_{L}-t_{0}=\alpha \log \frac{V_{\infty}-S}{V_{\infty}-m S} .
\end{aligned}
$$

Hence, $\Delta t$ increases with an increase of $\alpha$ and decreases with an increase of $V_{\infty}$, and in case $V_{\infty}$ is kept constant, there will be a certain liminal $\alpha$, above which no propagation can take place. This corresponds to the minimal gradient in the case of linearly rising current.

The general relation between $V_{\infty}$ and $\alpha$ for eliciting a propagated impulse is

$$
\Delta t=\alpha \log \frac{V_{\infty}-S}{V_{\infty}-m S} \leqq D .
$$

In the limiting case

$$
\begin{aligned}
& \alpha \log \frac{V_{\infty}-S}{V_{\infty}-m S}=D, \\
& \frac{V_{\infty}-S}{V_{\infty}-m S}=e^{D / \alpha} ; \quad V_{\infty}-S=e^{D / \alpha}\left(V_{\infty}-m S\right), \\
& V_{\infty}\left(e^{D / \alpha}-1\right)=S\left(m e^{D / \alpha}-1\right), \\
& \frac{V_{\infty}}{S}=\frac{m e^{D / \alpha}-1}{\boldsymbol{e}^{D / \alpha}-1}=\frac{m\left(\boldsymbol{e}^{D / \alpha}-1\right)+m-1}{\boldsymbol{e}^{D / \alpha}-1}, \\
& \quad=m+\frac{m-1}{\boldsymbol{e}^{D / \alpha}-1}=m+n \frac{1}{\boldsymbol{e}^{D / \alpha}-1}, \quad n=m-1 .
\end{aligned}
$$

If we take $D \fallingdotseq 1 \mathrm{msec}$, then

$$
\frac{V_{\infty}}{S}=m+n \frac{1}{e^{1 / \alpha}-1}, \quad \alpha, \text { in msec. }
$$

The graph of $1 /\left(\boldsymbol{e}^{1 / \alpha}-1\right)$ plotted against $\alpha$, is a beautiful straight line except in the range of very small $\alpha$ (fig. 11), that is, it very well fits the experimental data so far obtained (Solandt, Schriever, Suzuki, Sakaguchi and Tasaki (47-50)). The coefficient, $n$, which determines the slope is

$$
\begin{array}{ll}
n=m-1=e^{L / k}-1, & L: \text { minimal length, } \\
& k: \text { length constant. }
\end{array}
$$

Hence, the slope of the straight line is expected to increase with $L$ and to decrease with $k$.

The linear relationship above mentioned could also be derived from Hill's theory. The difference is that his is slightly concave downward and mine is slightly convex downward, in the region of very small $\alpha$. 


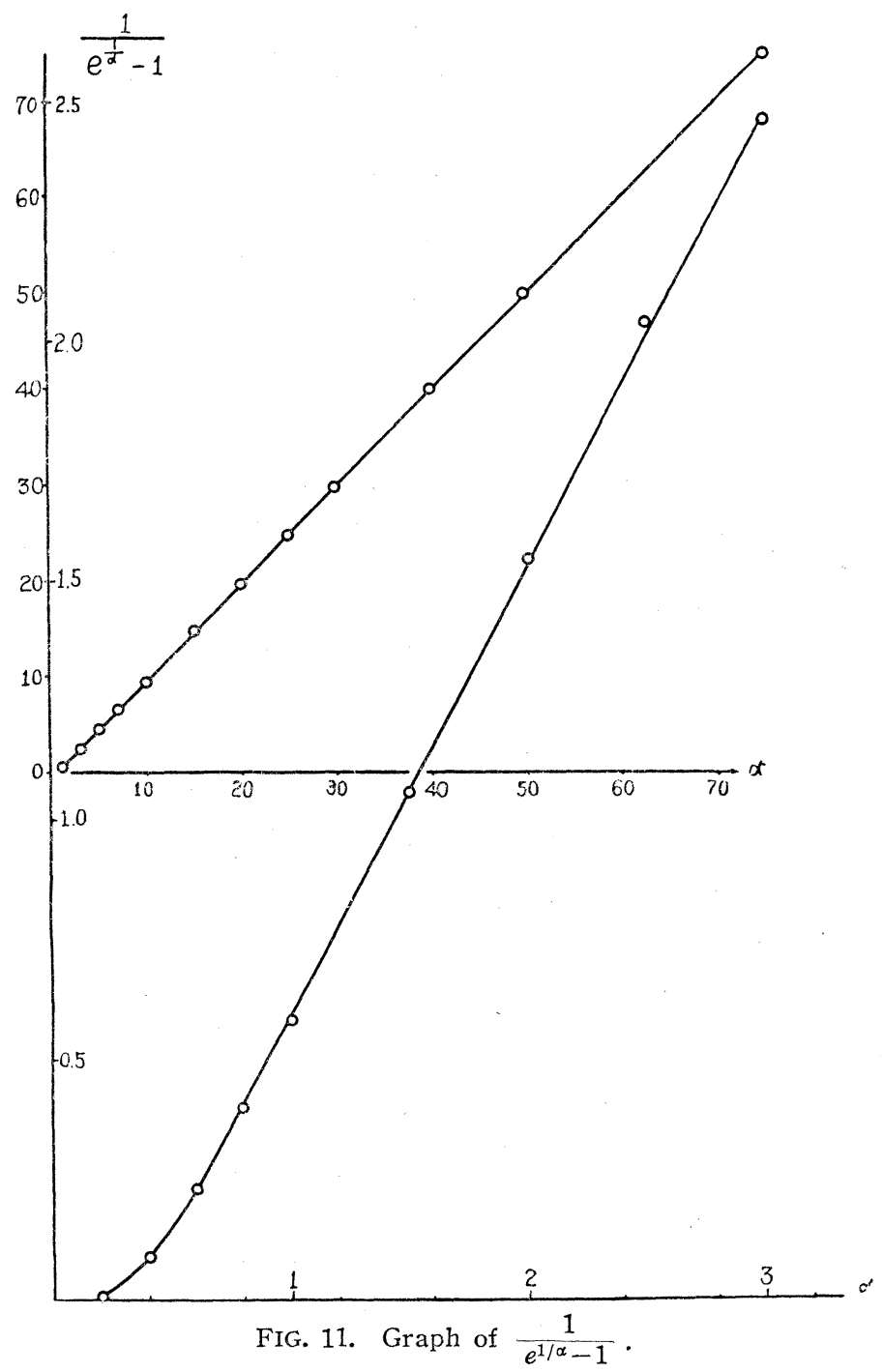

4. Repetitive responses to a long-lasting strong direct current We shall make here again the fundamental assumption,

$$
\begin{array}{ll}
P=V\left(1-e^{-t / \alpha}\right), & P: \text { local potential, } \\
& V: \text { applied voltage, }
\end{array}
$$

Then at points $x=0$ and $x=L$, we have as the condition for excitation

$$
\begin{aligned}
& x=0: P_{0}=V_{0}\left(1-e^{-t_{1} / \alpha}\right)=S, \\
& x=L: P_{L}=\frac{V_{0}}{m}\left(1-e^{-t_{2} / \alpha}\right)=S .
\end{aligned}
$$


The condition for propagation is, as before,

$$
t_{2}-t_{1}=\Delta t=\alpha \log \frac{V_{0}-S}{V_{0}-m S} \leqq D .
$$

In order that this relation can be established, $V_{0}$ cannot be smaller than a certain definite value, against a certain definite value of $\alpha$, and as long as $V_{0}$ is kept higher than this, propagation can always take place.

Now, what will happen after the first propagation? The local potential will disappear and begin to be recharged, but now the circumstances are not the same as before, because of the permeability change etc., and it seems very likely that $\alpha$ will increase, and accordingly $\Delta t$ will increase, too. Thus, propagation will be repeated with longer and longer intervals until it finally stops at the arrival of the moment, $\Delta t=D$. The whole time length during which the repetitive impulses can be elicited depends upon the largeness of $V_{0}$ applied and upon the smallness of $\alpha$-increase. Thus, we can understand very easily that 1 ), the interval between two successive impulses gets gradually longer, and 2), the repetitive impulses last for a certain definite time under a certain given $V_{0}$.

A point to be noticed in this consideration is that at the arrival of the moment $\Delta t=D$, propagation will stop, but the local excitation may not. This makes an essential difference from the classical ideas, where the stoppage of the impulse is explained by the rise of threshold (Adrian (51); Hill (38)), that is, by the stoppage of the excitation itself. Hill thinks, for example, that the local potential is charged up to a high value by the applied high voltage, and the repetitive impulses are elicited until the threshold gets as high as the local potential, by accommodation.

Whether local excitaion still occurs or not after propagation has stopped is a matter of experiment, but it is suggestive that in Lillie's nerve model I have often observed a repetitive activation which took place near the electrode only, by permanent application of a strong stimulus.

Finally, it is said that nerve fibres of small minimal gradients are liable to give repetitive impulses (Katz (52)). The smallness of the minimal gradient means, in the present theory, small $i_{r}$, large duration, small minimal length and large length constant (p. 143), which are all co-operative in establishing the relation $\Delta t<D$, so it will be natural that the fibres of small minimal gradients are liable to give rise to repetitive excitations.

\section{Electrotonus and excitability}

It is common knowledge that excitability is high in the cathodic region and low in the anodic region. The excitability here, is of course that for a propagated impulse, so it should be understood to be the reciprocal of the threshold, $V_{r}$, for exciting the minimal length, $L$, practically simultaneously. $V_{r}$ is expected to increase with the absolute value of $L$, and with $S$, the threshold potential for local excitation. Mathematically expressed:

$$
V_{r} \propto L \cdot S .
$$

As seen from eq. (4)' or fig. 14 (p. 153), $L$ decreases with increasing $k \log$ (S.F.), 
so we have, although roughly,

$$
L \propto \frac{1}{k \log (\text { S.F. })}, \quad \text { S.F. }=\frac{V}{S},
$$

Hence,

$$
V_{r} \propto \frac{S}{k \log (\text { S.F. })}
$$

It seems certain so far, but we do not know how $k, S$ and $V$ vary in electrotonus, and, consequently, we cannot see how $V_{r}$ will vary in actuality. We can only presume as we did before that the decrease of $S$ may be more effective than that of $k \log$ (S.F.), so that $V_{r}$ will become smaller in the case of catelectrotonus, at least as long as the tonus is weak or moderate (see the section "Electrotonus and V." p. 137).

Another known fact is the cathodic depression, that is, a reduction or loss of excitability in a strongly cathodic region. It is explained usually by a violent reduction of the action potential. The blocking phenomenon and the impossibility of impulse initiation can be understood by this, but the rise of $V_{r}$ and its effectiveness does not seem to be explained, without assuming accomodation which raises $S$ and, consequently, $V_{r}$. But if accomodation is really the case, why not in weak or moderate catelectrotonus?

Unfortunately, the present theory, too, is incapable of settling the question decidedly, but there is a way which seems promising; that is, the presumption that the decrease of $k \log$ (S.F.) becomes dominant as compared to that of $S$, contrary to the case of weak or moderate catelectrotonus. The result will be the enlargement of $V_{r}$, even to infinity.

In very strong catelectrotonus, further, I suppose that spontaneous repetitive local excitation will occur near or about the electrode. The artificial as well as conducting stimuli will then be ineffective, because they may fall well within a refractory period.

6. Interrelation among electrode distance, applied voltage, length corstant and minimal length

It is known that the electrode distance stands in intimate relation with the applied voltage for obtaining a certain definite excitation (Rushton (53)). If viewed from the present theory, the length constant, which defines the electrotonic current spread, and the minimal length necessary for impulse initiation, also play, a role which will be shown in the following, putting aside the time factor for simplicity.

In fig. 12, the points $M$ and $N$ represent the two points where just the threshold potential governs, i.e.,

$$
P_{M}=P_{N}=S \text {. }
$$

Now,

$$
\begin{aligned}
& P_{M}=P e^{-x / k}-P e^{-(X-x) / k}=S, \\
& e^{-x / k}-e^{-(X-x) / k}=\frac{S}{P},
\end{aligned}
$$




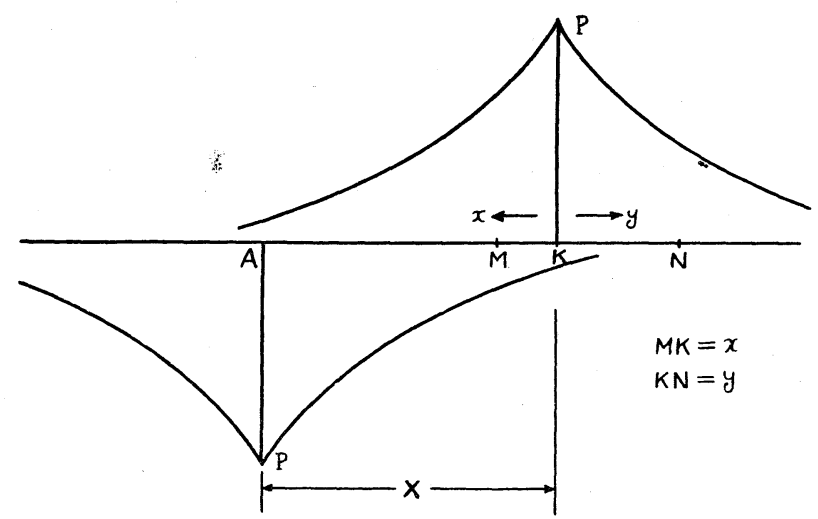

FIG. 12. Distribution of anodic and cathodic potentials.

$M, N$ : Points of just threshold potential. $X:$ Electrode distance.

For $X=\infty$ :

$$
\begin{aligned}
& e^{-x / k}=\frac{S}{P}, \\
& x=k \log \frac{P}{S}, \\
& 1-e^{-X / k}=\frac{S}{P}, \\
& e^{-X / k}=\frac{P-S}{P}, \\
& X=k \log \frac{P}{P-S} .
\end{aligned}
$$$$
\text { For } x=0 \text { : }
$$

Hence, $x$ increaese from zero to $k \log \frac{P}{S}$ in accord with $X$ the change from $k \log \frac{P}{P-S}$ to infinity.

Similarly, about the point $N$,

$$
\begin{aligned}
& P_{N}=P e^{-y / k}-P e^{-(x+y) / k}=S, \\
& e^{-y / k}-e^{-(x+y) / k}=\frac{S}{P},
\end{aligned}
$$

For $X=\infty$ :

$$
\begin{aligned}
& e^{-y / k}=\frac{S}{P}, \\
& y=k \log \frac{P}{S},
\end{aligned}
$$

For $y=0$ :

$$
\begin{aligned}
& 1-e^{-X / k}=\frac{S}{P}, \\
& e^{-X / k}=\frac{P-S}{P}, \\
& X=k \log \frac{P}{P-S} .
\end{aligned}
$$


Hence, $y$ increases from zero to $k \log \frac{P}{S}$ as $X$ varies from $k \log \frac{P}{P-S}$ to infinity, just as $x$ does.

Now, subtracting eq. (4) from (1), we have

where

$$
\left(e^{-x / k}-e^{-y / k}\right)-\left(e^{-(X-x) / k}-e^{-(X+y) / k}\right)=0,
$$

$$
\begin{aligned}
& e^{-(x-x) / k}>e^{-(x+y) / k}, \\
\therefore \quad & e^{-x / k}>e^{-y / k}, \\
\therefore \quad & x<y .
\end{aligned}
$$

Hence, we have rough pictures of $x$ and $y$ in relation to $X$, as represented by fig. 13. In order to have a propagation, we have to have

$$
x+y \geqq L, \quad L \text { : minimal length. }
$$

To meet this requirement, $X$ must be varied inversely to $P$ in proper magnitude, as seen directly from fig. 13.

Further, in case $X=\infty$,

$$
\begin{gathered}
x+y=2 k \log \frac{P}{S}=L, \\
\frac{P}{S}=e^{L / 2 k}, \\
P=S e^{L / 2 k} .
\end{gathered}
$$

In words, $P$ must be $e^{L / 2 k}$-times larger than $S$, even in case $X=\infty$. This is different from the usual idea, where the relation $P=S$ is required.

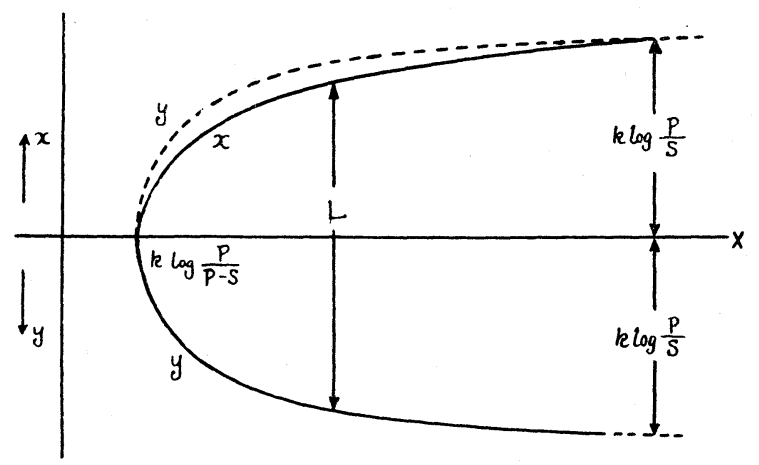

FIG. 13. $y$ and $x$ in relation to $X$ (refer to fig. 12 ).

\section{Latency}

It is long known that there is a somewhat latent period between the end of the stimulating pulse and the appearance of the "spike" potential, particularly clearly in the case of brief threshold stimulation (Blair and Erlanger (54)). The existence of the period in question was confirmed recently by Rosenblueth and Luco (31), Maruhashi (55) and Imamura (56). The measurement of the time 
intervening between the onset of the stimulus and the appearance of the action potential-the so-called "response time"-was attempted, too, by Tasaki and Fujita (57) and Imamura (56), which, according to Imamura, involves the latent period as a part.

According to Imamura, the latency is shortened by higher voltage as well as longer duration of the stimulating current, while, in case the current is cut off just at its utilization time, the latency fluctuates very much and shows no regular relation to the voltage. But, at least as long as the pulse is made so brief that the action potential appears always after the break, latency appears certainly lengthened in lower voltage, until finally it becomes fairly long at or near threshold stimulation.

The existence of latency has thus become certain, but its nature is still quite vague. It may be a period, during which some special physiological process - say, "excitatory process (Imamura (56))"-is going on, or it may be something else, for example, the conduction time in a node of Rauvier (Moruhashi (55)). I will show here that there is a way of understanding it from the present theory, too.

It should be remarked beforehand that 1) recent observations were made on single nodes of Ranvier directly stimulated, but the index employed was the spike and not the local response, so that it was equivalent to employing the propagated impulse as an index; 2) the spike appears, after the present theory, first when the initial small action length develops suddenly into a large one (perhaps into sudden occupation of a whole node in the case of myelinated fibre), with or without help from the stimulating current; and 3) the latency hitherto measured and considered is that for the spike, but not for the local excitation.

Now we take the so-called latency intervening between the end of the utilization time and the appearance of the spike, as nothing but the utilization time of the action current originating from the initial action length $L$, which is spent for producing the next large action length. In case the stimulating current persists, it will co-operate with the action current, to shorten the latency. This is the very point which is quite different from the classical way of consideration.

\section{1) The case where pulse duration is made equivalent to utilization time}

In this case, the stimulating current is out of action at the end of the utilization time, so the action current from the length $L$ is the only stimulus acting on its neighbour. Hence, the latency as defined above is expected to be approximately constant without regard to the applied voltage. Strictly speaking the excitation of $L$ cannot be quite simultaneous, but is completed in $\Delta t$, which is longer in weaker stimulation, as mentioned repeatedly. Hence, the action current flowing out of $L$ as a whole, will be flatter, less intensive and longer lasting in weaker stimulation, and, consequently, the utilization time, that is, the latency, will be lengthened a bit. If so, the latency will show a small regularity, that is, a gradual lengthening with weaker applied voltage.

The above mentioned experiment was carried out by Imamura. He encountered, however, a great fluctuation of the latency as Blair and Erlanger (54). 
and Maruhashi (55) did, and could not find any definite relation to the applied voltage. This result may be taken as indicating the practical independence of the latency from the voltage, or the small regularity expected which, however, was disturbed by the large fluctuation, whatever the cause of the fluctuation may be.

\section{2) The case of constant pulse duration}

In this case, the stimulating current flows on a little while after the utilization time is over, and co-operates with the action current from $L$ for exciting its neighbour. This surplus time increases with voltage, because the utilization time decreases with voltage. Therefore, the effect of co-operation will become stronger in higher voltage, which will be manifested in shortening the latency.

This theoretical expectation is just what was actually found in Imamura's experiment. The fluctuation of the latency might have prevailed in this case, too, but perhaps it was covered by the great regularity brought about by the stimulating current co-existing.

\section{3) The case of constant voltage of various durations}

From the same reasoning as above, a shorter latency is expected theoretically in longer duration. And it was just the case, too, in Imamura's experiment.

In the above argument, it was not questioned whether or not there exists a latency in the true sense at the directly stimulated site itself. What was shown above is that, even if there is none at the stimulated point, still the so-called latency can appear and that in intimate relation to the applied voltage, if only the full-sized spike or the conducted impulse is taken as the index.

\section{THE STANDARD $y$ - $x$-RELATION, THE VALUES OF $l, L$ AND $M$, AND SOME CONSIDERATIONS ABOUT MYELINATED NERVE FIBRE}

An exact graphical representation of eq. (4) (p. 132) is given in fig. 14, assuming $k$ (length constant) $=5$ and (Safety factor) $=5$ and 10 . Here we see that the nerve in physiological conditions belongs actually to the type of fig. 3 , I, and that a minute initial action length develops into an enormous one in the next stage. The values of $l, L$ and $M$ in $\mathrm{mm}$. are respectively $0.21,0.22$ and 8.05 in the case of S.F. $=5$ and $0.10,0.11$ and 11.57 in the case of S.F. $=10$. The most important length $L$, which was $0.86 \mathrm{~mm}$. in Rushton's theory, turned out to be about 4-8-times shorter in the present theory.

Whether the specific length, $L$, exists in the myelinated fibre or not, may be quite a different matter. If judged simply from the figure obtained above, it is clear that it cannot, because a node of Ranvier is said to be $1 \mu$ or less wide. But it is note-worthy that Tasaki's result quoted on p. 153 can give us $L \fallingdotseq 5 \mu$, if combined with Rushton's data $k=5 \mathrm{~mm}$. (2). This is suggestive of a possibility of $L$-existence in a node of Ranvier. It is as follows :

Fig. 15 is a rough reproduction of Tasaki's result (44). The line $A C$ represents the minimal gradient, and the line $A F$ the current flowing at the point $x=L$. Now, in order for a propagation to take place, the excitation at $L$ must occur by the end of $D$ (excitation duration) of the point $x=0$. Hence, in fig. 


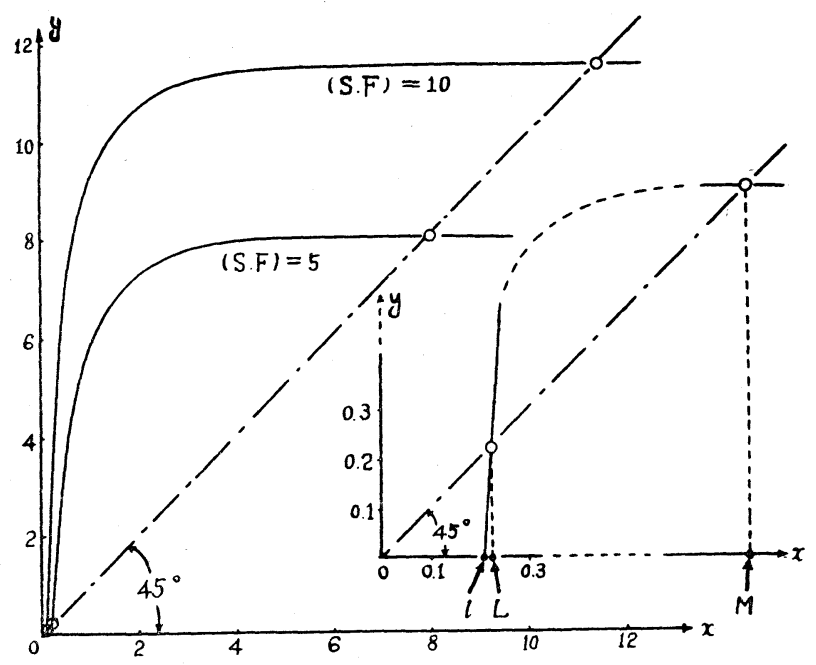

FIG. 14. Standard $y$-x-relation.

15, $C F$ must be smaller than or equal to $D$, because it denotes the time difference, $\Delta t$, of excitations of the points $x=0$ and $x=L$.

$$
\begin{gathered}
\text { Now, } \quad A B: C F=B E: C E, \\
A B: A B+C F=B E: B E+C E \\
=B E: B C
\end{gathered}
$$

$C F(=D)$ may be taken about 1 msec., while $A B$ varied widely from several tens to several hundreds of msec. in Tasaki's experiment. If assumed that $A B$ can attain a 1,000 msec., we have

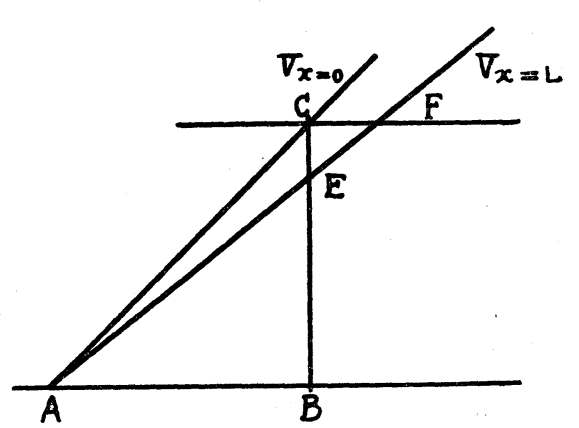

FIG. 15. Evalution of $L$.

$V_{w=0}$ : Current of min. gradient.

$$
A B: A B+C F=1,000: 1,001=B E: B C,
$$

Now, $B E / B C$ is the ratio of the currents flowing at $x=L$ and $x=0$, hence, $B E / B C=i_{x=L} / i_{x=0}=e^{-L / k}=\frac{1,000}{1,001}$, where $k$ is the length constant.

$$
\begin{aligned}
\therefore \quad \frac{L}{k} & =\log \frac{1,001}{1,000} \fallingdotseq 0.001, \\
L & \fallingdotseq 0.001 \times k .
\end{aligned}
$$

According to Rushton (2) $k \fallingdotseq 5 \mathrm{~mm}$., so we have $L \fallingdotseq 5 \mu$. This is only a rough approximation, but it is interesting to note that the value obtained is not far from the order of the nodal breadth. I do suppose thus, that in actuality, $L$ might be a fraction of a nodal breadth, at least in the case of myelinated fibres. 
This is the very reason why I quoted a few works concerned with single nodes of Ranvier in the preceding chapter, and discussed them from the same standpoint.

Based on this, I imagine a picture of a Ranvier's node as illustrated in fig. 16, where $L$ denotes the position of the point $x=L$, supposed to exist. Let $P_{0}$ be the potential evoked at the left edge of $N_{1}$ by the action current from $N_{0}$. The potential at $x=L$ will be

$$
P_{L}=P_{0} e^{-L / k}
$$

If $P_{L}>S$, then a conduction will occur in $N_{1}$ to occupy the whole nodal breadth. If $P_{l}>S$, then the whole $N_{1}$ will get excited practically simultaneously, without waiting for conduction. Perhaps this is the usual occurrence. At any rate, the excitation of the whole $N_{1}$ will give the so-called full-sized spike, which will provoke $P_{0}$ at the left edge of the next node $N_{2}$, and so on. If $P_{0}$ becomes so low that $P_{L}<S$ for some reason or other, then the excitation will be limited in the region $O L$, which will result in giving a local response. The same can be said about artificial stimulation, unless some special method like Marmont's (60) is employed.

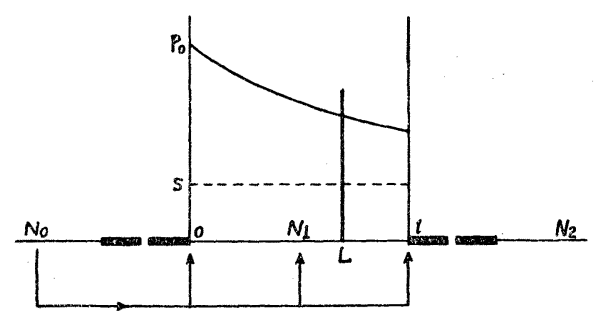

FIG. 16. Outward action current at node, $N_{1}$. $N_{0}, N_{1}, N_{2}$ : Nodes of Ranvier

$P_{0}$ : Potential at 0 , established by the action potential of $N_{0}$

$S$ : Threshold

$L$ : Point of min. length, supposed to exist.

There is, however, a point to be questioned in the above consideration, that is, the reason why such a large difference appears between the two values of $L$ obtained in two different ways, as described above. It seems to be too large to be attributed to the neglects in mathematical treatment, or to the inexact values of physiological constants available at present. It might be partly due to the fact that the safety factor was made too small in evaluating $L$ from fig. 14, because it should have been the safety factor for a local excitation, but still it seems too great to explain the large discrepancy encountered. Is there then some essential difference between myelinated and unmyelinated fibres? Nothing can be said decisively at present. But at any rate I should think that as far as myelinated fibres are concerned, we should rely on the experimental data obtained directly from that tissue, and on the theoretical deductions derived therefrom. Thus I believe in the correctness of the value of $L$ estimated above as that of myelinated fibres.

\section{DISCUSSION}

\section{On the interpretation of the local response}

There are three opinions about this, as stated elsewhere. Hodgkin et al. 
regarded it first as an event occurring in a region smaller than minimal length, but later took it as a smaller activity of one and the same region (58), after reconfirmation (59) of the works of Marmont (60) and Cole (34). These two opinions are different in the sense that one refers to the space and the other to the intensity, but they are the same in believing the identity of the local response and the spike. On the other hand, Rosenblueth et al. claim that these two are quite different in nature as well as in properties (36).

Hodgkin (58) states that Marmont (60) and Cole (34) stimulated a definite area $\left(6 \mathrm{~mm}^{2}\right)$ of nerve membrane employing Marmont's new method and obtained subthreshold responses with weak stimuli. They made the condition of the area strictly uniform so as no variation could occur in the area activated. But, could such a large area be uniform, physically and physiologically, in the scale of $L$ which might have been of the order of $0.1-0.01 \mathrm{~mm}$. or less? If not, there is a possibility that "islands" of excitation appear in weak stimulation, to be caught as subthreshold excitation of the whole area.

On the other hand, Rosenblueth points to the following facts as the basis of his claim (36): 1) the local response does not follow the all-or-nothing, 2) there is no threshold, 3) summation occurs, 4) local response and spike can appear separated in the record, and 5) under anodic polarization, spike appears larger than normal, while local response smaller. Of these, 5 ) is really puzzling, but the other four are not unexplainable from the spatial idea, i.e., 1) the potential on the fibre surface increases with increase of the active area (Rushton $(2)), 2)$ a smaller area responds to a weaker stimulus, 3) a new excited area is added by co-operation of the second stimulus and the action current from the first excited area, and 4) spike is evoked, in the present theory, by the action current originating from the initial action length and acting on the neighbouring region, at least in the case of threshold stimulation. In this case, the moment of spike-appearance comes after the "effect" of the local response has attained a threshold value, accordingly, it can be after the peak of the local response. Actually, Rosenblueth observed such a case, so did Ichioka in our laboratory (unpublished). This phenomenon seems to point to something which is being stimulated by the local response. It can be the adjacent region of the fibre, if viewed from the spatial theory, but very difficult to imagine from the other two.

Thus, it seems right to me to take the local response in the spatial sense, because, although there is no positive proof, the possibility is large and, moreover, it helps us understand equivocally a number of fundamental phenomena of excitation and conduction, as described above.

\section{On the laws of excitation in general}

It was claimed in this argument that a certain minimal length must always be excited for propagation to occur. If this is true, then it is clear that any law of excitation obtained by employing the propagated impulse as index, cannot always be a law at the stimulated point. The point is whether excitation and conduction is identical or not. At any rate, the two laws should be worked out separately.

However, they are not quite disconnected, either. For example, the $i-t$-rela- 
tion hitherto worked out is nothing but the relation between the current $i$ flowing at the point $x=0$ and the utilization time explored at the point $x=L$, where a current $i / m$ is flowing. Hence, if we plot $i / m$, instead of $i$, against $t$, then the whole curve represents the local $i$ - $t$-relation at the point, $L$. The value of $m$ is not quite clear, but certainly $m$ is larger than and very near, unity, because $1 / m=e^{-L / k}$, where $L$ is the minimal length and $k$ the length constant. Hence, $i / m$ - $t$-relation will be a little flat as compared to $i$-t-relation, but it keeps the original shape as it is. Of course, it should be born in mind that in the actual experiments the range of $t$ was limited definitely by the condition $\Delta t \leqq D$, and consequently any extrapolation is nonsense.

\section{The all-or-nothing principle}

We have been convinced of the all-or-nothing principle of the nerve fibre as one of the best established acquisitions. The finding of the local response taught us the existence of another type of nervous activity which does not follow the principle. If, now, the intensity theory of the local response is correct, then the all-or-nothing principle is evidently destinated to be thrown away after all, leaving behind a difficult question, how a sudden jump occurs when the local response has attained a certain threshold value. The only way to rescue the principle in this case is to regard the spike only as the excitation and to regard the local response as something else, as Rosenblueth did; for example, as some preparatory process, say, the stimulatory process. But if so, the fact that the spike can appear after the local response has substantially subsided from its peak, turns out to be a problem difficult to understand.

In the spatial theory, on the other hand, the all-or-nothing principle is kept on as it has been, because the principle is assigned to the final excitable element from the beginning in this theory. In this case, however, we have also to note that 1) the "all" is an assumption after all, 2) what we can catch actually is graded, and 3) what we have caught as "all" is nothing but the "largest" attained by group of elements, and thus we may have perhaps to distinguish the true from the apparent all-or-nothing principle.

\section{A doubt about the concept of "accommodation"}

The arguments in chapter $\mathrm{V}$ were all developed without introducing the concept "accommodation." The basic assumption made was very simple and probable, though not exact quantitatively, and yet a number of fundamental phenomena of conduction and excitation could be explained. Of course, present reasoning, by itself, cannot deny accommodation, but only shows a possibility of explaining or understanding the experimental facts without taking it into consideration. But Sugi's result and the horizontal part of Tasaki's and Diecke's results quoted on p. 143 seem to offer contra-evidences against accommodation. Diecke, too, confirmed the minimal gradient, but interpreted it differently and denied the classical accommodation defined by rise in threshold. Here arises a slight doubt about the concept: was it not a faulty concept deriving from mistaking the propagated impulse as the index of the local excitation?

The final decision must be await observations made directly on excitation at the stimulated locus, and that on the local response of a certain definite 
magnitude.

\section{Supposition of a physiological unit of action length}

All the argument hitherto developed on excitation, including the present one, tacitly assumed the possibility of excitation in an infinitesimal area, expressed vaguely by the word "point." As long as a point excitation in this sense is granted, and as long as there is a certain threshold current for exciting a point, a certain definite area is unavoidably required to be excited in order that propagation can take place, because otherwise the local current will be infinitesimally weak. This is perhaps the very reason why the minimal length $L$ appeared in Rushton's and in the present treatment.

Now, what will become of the whole picture, if it is assumed that there is a certain definite length or area as the excitable unit in the physiological sense? Then, even in case just one unit is excited, the local current can be finite, provided that the local resistance and the action potential are given in proper magnitudes, and the unit-to-unit conduction, formally just similar to the pointto-point conduction, is not impossible. Excitation and conduction can then become identical. The nodal breadth might turn out to be nothing but this unit. The whole picture of conduction and its theoretical treatment might change enormously.

This is only an imaginative picture at present, but at any rate it seems to be unwise to think of a "point" vaguely in every actual case of stimulation and conduction. The definition or exploration of the unit may be essential in future research. There is room here for another important phase of precise observation on the directly stimulated locus.

\section{SUMMARY}

1. The fibre length $y$ which can be excited by an action length $x$ was calculated. The chief results obtained were as follows:
1) $l>x: y=0$
$l:$ a small definite length
2) $l<x<L: y<x$
$L$ : another definite length larger than $l$
3) $L<x<M: y>x$,
$M$ : another definite length larger than $L$
4) $x>M: y<x$,

2. Standing on the above results, it was argued that there can arise five types of conduction with regard to action length, action current and conduction velocity, as follows:

1) Local graded response (no conduction),

2) Local graded conduction (decremental),

3) Constant conduction,

4) Incremental-constant conduction,

5 ) Decremental-constant conduction.

3. The length, $L$, was defined as the minimal length necessary for initiation of impulse. Based on this, it was stressed that conduction is, in essence, from length to length, although apparently from point to point or continuous.

4. The following phenomena of conduction were explained from $y$-x $x$-relation above stated: 
1) Local graded responses and conduction,

2) Conduction in narcotized region (decremental-constant, decremental or local),

3) Larger velocity in higher temperature,

4) Dependence of velocity upon stimulation intensity and distance travelled,

5) Larger velocity of thicker fibres,

6) Larger velocity under cathode and smaller velocity under anode.

5. Several excitation laws obtained with conducted impulses as index, were interpreted as those for just exciting the length, $L$, and from this view-point, some well-known excitation phenomena were explained:

1) Appearance of rheobase and "Hauptnutzzeit."

2) Appearance of the minimal gradient of the linearly increasing current.

3) Phenomena in stimulation with exponentially increasing current.

4) Repetitive responses to strong, long-lasting constant current.

5) Excitability change under electrotonus.

6) Interrelation among electrode distance, applied voltage and length constant at bipolar stimulation.

7) Latency of the action potential.

6. Evaluation of the length, $L$, was attempted. It appeared to be of the order of $1 \sim 100 \mu$.

7. Discussions were made on the following thema:

1) Interpretation of the local response.

2) Excitation laws in general.

3) All-or-nothing principle.

4) "Accommodation."

5) Fundamental unit length of action.

8. It was stressed that the laws of local excitation and of conducted impulse should be worked out separately.

Cordial thanks are due to my collaborators in the laboratory who kindiy helped me during illness in preparing the manuscript, particularly to Mr. S. Tsukui for numerical calculations and graph drawings.

\section{REFERENCES}

1. Yamagiwa, K. J. Jap. Physiol. Soc. $5: 1,1940$ (Japanese).

2. Rushton, W. A. H. Proc. Roy. Soc. B. $124: 210,1937$.

3. Hodgkin, A. L. J. Physiol. $106:$ 305, 1947.

4. Rushton, W. A. H. J. Physiol. 82 : 352, 1934.

5. YAmAgIWA, K. The Builetin of Tokyo Med. and Dent. Univ. 1: 133, 1954.

6. TASAKI, I. Amer. J. Physiol. 127 : 211, 1939.

7. TASAKI, I. AND MizuguchI, K. Biochim. et Biophys. Acta. 3 : 484, 1949.

8. TASAKI, I. Nervous Transmission, p. 92. Springfield, U.S.A. : Charles C. Thomas, 1953.

9. LUCAS, K. The conduction of nervous impulse. London: Longmans, Green and Co. London, 1917.

10. KATO, G. The theory of decrementless conduction. Tokyo: Nankodo, 1924.

11. Tasaki, I. AND Takeuchi, T. Pflüg. Arch. ges. Physiol. $244:$ 696, 1941. 
12. Hodgkin, A. L. J. Physiol. $108: 37,1949$,

13. Engelmann, Th. W. Pflüg. Arch. ges. Physiol. 66: 574, 1897.

14. Nicolai, G. F. Pflüg. Arch. ges. Physiol. $85: 65,1901$.

15. TASAKI, I. Nervous Transmission. p. 41, 128. Springfield, U.S.A.: Charles C. Thomas, 1953.

16. YAMAgIWA, K. Jap. Med. J. $1: 439,1948$.

17. FISCHL, E. AND KAHN, R. H. Pflüg. Arch. ges. Physiol. 219 : 32, 1928.

18. Pratt, R. H. Amer. J. Physiol. 93 : 680, 1930.

19. Brown, D. E. S. AND Sichel, O. F. J. M. Science $72: 17,1930$.

20. Gelfan, S. Amer. J. Physiol. 93 : 112 ; $96: 16,1930-31$. J. Physiol. $80: 285,1934$.

21. Gelfan, S. And Gerard, R. W. Amer. J. Physiol. $95: 412,1930$.

22. Gelfan, S. AND Bishop, G. H. Amer. J. Physiol. $101: 678 ; 103: 237,1932-33$.

23. HASHIDA, K. Jap. J. med. Sci. III, Biophysics 2: 35*, 1931.

24. Asmussen, E. Pflüg. Arch. ges. Physiol. 230 : 263 ; Skand. Arch. 65 : 261, 1932-33.

25. KATz, B. Proc. Roy. Soc. B. $136: 506,1948$.

26. Hodgkin, A. L. Proc. Roy. Soc. B. $126: 87,1938$.

27. Pumphrey, R. J., Schmitt, O. H. AND Young, J. Z. J. Physiol. 98 : 49, 1940.

28. Hodgkin, A. L. And Rushton, W. A. H. Proc. Roy. Soc. B. 133 : 447, 1940.

29. KATZ, B. J. Physiol. 106: 66, 1947.

30. Huxley, A. F. AND Stämpfli, R. J. Physiol. 112: 476, 1951.

31. Rosenblueth, A. ANd Luco, J. V. J. cell. comp. Physiol. $36: 289,1950$.

32. Rosenblueth, A And Ramos, J. G. J. cell. comp. Physiol. $38: 321 ; 39$ : 109, 1951-2.

33. Del Castillo-Nicolau, J. AND Stark, L. J. Physiol. 118: 207, 1952.

34. Cole, K. S. Arch. Sci. Physiol. 3: 253, 1949. Quoted from Hodgkin (58).

35. ICHIOKA, M. Kagaku 24: 150, 1954 (Japanese).

36. Rosenblueth, A. Ergebn. Physiol. 47 : 56-57, 1954.

37. OzORIO DE ALMEIDA. Ann. physiol. et physico-chim. biol. 3: 129, 1927.

38. Hill, A. V. Proc. Roy. Soc. B. 119: 305, 1936.

39. LApicque, L. J. Physiol. et Path. gén. 9: 620, 1907.

40. Rushton, W. A. H. J. Physiol. 72: 265, 1931.

41. LUCAS, K. J. Physiol. $36: 253 ; 37: 459,1907-8$.

42. FABRe, PH. C. R. Acad. Sci., Paris 184:699, $1486 ; 185: 300,1216,1927$.

43. TASAKI, I. Biochim. biophys. acta 3: 498, 1949.

44. TASAKI, I. Jap. J. Physiol. 1: 1, 1950.

45. TASAKI, I. The physiology of the nerve flbre, p. 210. Tokyo: Kawai, 1944 (Japanese).

46. SugI, Y. Jap. J. Physiol. Soc. 4: 95, 1939 (Japanese).

47. Solandt, D. Y. Proc. Roy. Soc. B. $119:$ 355, 1936.

48. SCHRIEVER, H. Z. Biol. 91 : 173, 1930.

49. Suzuki, M. Pflüg. Arch. ges. Physiol. $230: 363 ; 239: 81,1932-37$.

50. SAKAguchi, K. AND TASAKI, I. Jap. J. Physiol. 1: 7, 1950.

51. ADRIAN, E. D. Physiol. Rev. 10: 336, 1930.

52. KATZ, B. J. Physiol. 88: 239, 1937.

53. Rushton, W. A. H. J. Physiol. $63: 357,1927$.

54. Blair, H. A. ANd Erlanger, J. Amer. J. Physiol. $106: 524 ; 144: 309,1933-36$.

55. MARUHASHI, J. Keio Igaku 29: 187, 1952 (Japanese).

56. Imamura, I. Coliected Papers, Physiol. Lab., Showa Med Univ. 6: 15, 1954.

57. TASAKI, I. AND FUJITA, M. J. Neurophysiol. 11: 311, 1948.

58. HodGKIN, A. L. Biol. Rev. $26: 339,1951$.

59. Hodgkin, A. L., Huxley, A. F. ANd KATZ, B. Arch. Sci. physiol. 3 : 253, 1949.

60. MARMONT, G. J. cell. comp. Physiol. 34: 351, 1949.

61. DIECKE, F. P. J. Federation Proc. $13: 36,1954$. 